\title{
Magnetic resonance imaging in lumbar gunshot wounds: an absolute contraindication?
}

\author{
Eduardo Martinez-del-Campo, M.D., Leonardo Rangel-Castilla, M.D., \\ Hector Soriano-Baron, M.D., and Nicholas Theodore, M.D.
}

Division of Neurological Surgery, Barrow Neurological Institute, St. Joseph's Hospital and Medical Center, Phoenix, Arizona

\begin{abstract}
Object. Performance of MR imaging in patients with gunshot wounds at or near the lumbar spinal canal is controversial. The authors reviewed the literature on the use of MR imaging in gunshot wounds to the spine. They discuss the results from in vitro and clinical studies, analyze the physical properties of common projectiles, and evaluate the safety and indications for MR imaging when metallic fragments are located near the spinal canal.

Methods. A review of the English-language literature was performed. Data from 25 articles were analyzed, including 5 in vitro studies of the interaction between 95 projectiles and the MR system's magnetic fields, and the clinical outcomes in 22 patients with metallic fragments at or near the spinal canal who underwent MR imaging.

Results. Properties of 95 civilian and military projectiles were analyzed at a magnet strength of $1,1.5,3$, and $7 \mathrm{~T}$. The most common projectiles were bullets with a core of lead, either with a copper jacket or unjacketed (73 [76.8\%] of 95). Steel-containing (core or jacket) projectiles comprised $14.7 \%$. No field interaction was evident in 78 (96.3\%) of the 81 nonsteel projectiles. All steel projectiles showed at least positive deflection forces, longitudinal migration, or rotation. Heating of the projectiles was clinically insignificant. Image artifact was significant in all 9 steel bullets tested, but was not significant in $39(88.6 \%)$ of the 44 nonsteel bullets tested. Overall, 22 patients with complete $(82 \%)$ and incomplete $(14 \%)$ spinal cord injury secondary to a projectile lodged inside the spinal canal underwent MR imaging. Discomfort and further physical or neurological deficits were not reported by any patient. Two patients with spinal cord injuries underwent MR imaging studies before surgical decompression and had subsequent, significant neurological improvement.

Conclusions. Metallic implants near or at the spinal canal are a relative contraindication for MR imaging. However, safe MR imaging might be feasible when a projectile's properties and a patient's individualized clinical presentation are considered.
\end{abstract}

(http://thejns.org/doi/abs/10.3171/2014.7.FOCUS1496)

KeY Words • bullet • gunshot • lumbar • magnetic resonance • spinal

$\mathrm{G}$ UNSHOT wounds (GSWs) account for 13\%-17\% of spinal cord injuries (SCIs) in young males (ages 15-34 years). ${ }^{4,15,17,26} \mathrm{~A}$ person with a GSW at or near the spinal canal is likely to have a complete SCI with a poor prognosis. ${ }^{4}$ Magnetic resonance imaging is sometimes necessary to complete an evaluation of a patient with an SCI, ${ }^{5}$ because MR imaging identifies damage to ligaments, the spinal cord, and nerve roots. ${ }^{2,4,15}$

For decades, MR imaging has seldom been used for people with GSWs. . $^{13,17,23}$ This is because of concerns about the magnetic field's effect on ferromagnetic bullet components that could potentially exacerbate the original injury. Therefore, GSWs have been considered a contraindication for MR imaging. ${ }^{23}$ Studies that have looked into these interactions have drawn different con-

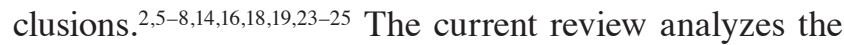
most recent evidence available in the literature to deter-

Abbreviations used in this paper: $\mathrm{GSW}=$ gunshot wound; $\mathrm{SCI}=$ spinal cord injury. mine the role of MR imaging in patients with a GSW to the lumbar spinal canal.

\section{Methods}

A review of English-language literature was performed on PubMed using the key words "magnetic resonance," "lumbar," "spine," "gunshot," "bullet," and "metal." Additional articles were located by cross-referencing articles encountered initially through PubMed searches. Inclusion criteria comprised articles (case reports, case series, literature reviews, and guidelines) published in the peer-reviewed literature; these articles described in vitro and in vivo studies, with tests addressing the radiological and physical interactions between magnetic fields and bullets or magnetic fragments. Thirty-eight articles were reviewed, and 25 were selected for inclusion based on the criteria listed above.

Data collected from these 25 studies included projectile properties, such as ferromagnetism, geometrical 


\section{E. Martinez-del-Campo et al.}

shape, and core and jacket composition. Projectile interactions with the MR system's magnetic field were also analyzed, such as deflection force (positive if $\geq 45$ dynes), longitudinal migration, deflection angle (rotation), heating, and image artifact. Image artifact (black void and image distortion due to local perturbations in the static magnetic and gradient fields) was classified as none (indistinguishable); mild (+, same size as the projectile); moderate (++, larger size than the projectile but with insignificant image distortion); and severe (+++, markedly larger than the object and with image distortion).

Data gathered from clinical studies and case reports of single patients undergoing MR imaging included management of patients with GSWs; location of the bullets; clinical indications for MR imaging; type of MR imaging used; control CT imaging before and after MR imaging; time between injury and MR imaging; and, if available, longitudinal migration and rotation of the bullet/bullet fragments as well as the patients' neurological status changes and self-reported symptoms. Surgical decompression, when needed, was also reported. The publication dates of the 25 articles ranged from 1985 to 2014 .

\section{Results}

\section{In Vitro Studies}

Information on interactions between bullets and the MR imaging system's magnetic field was collected from 5 in vitro studies (Table 1). ${ }^{5,12,16,22,25} \mathrm{~A}$ total of 95 hunting, civilian, police, and military projectiles were analyzed in MR scanners with magnetic strengths of $1,1.5,3$, and 7 T. Eighty-eight bullets were made in the US (domestic), and 7 were from 6 different countries (foreign). ${ }^{25}$ Eightyseven projectiles were bullets and 8 were pellets. The most common projectile composition was lead core/copper jacket (43 [45.3\%]), followed by lead core/unjacketed (30 [31.6\%]). Only 14 projectiles (14.7\%) were composed of steel (9 steel-core bullets, 4 steel-core spherical projectiles, 1 steel-jacket bullet).

Almost all (78 [96.3\%] of 81) nonsteel projectiles were not ferromagnetic and showed no migration, rotation, or deflection after MR imaging. Interestingly, 3 (3.7\%) of 81 nonsteel projectiles that showed rotation and deflection force were of foreign origin. Overall (steel and nonsteel), 6 bullets rotated $90^{\circ}$ and 6 bullets rotated less than $90^{\circ}$ (between $45^{\circ}$ and $89^{\circ}$ ). Two steel pellets showed a "potential" for rotation, as reported by the authors. ${ }^{16}$ Only bullets perpendicular to the $\mathrm{z}$ axis showed rotation, whereas bullets parallel to the $\mathrm{z}$ axis did not. Most spherical projectiles (6) showed no rotation, regardless of their material composition, whereas 2 steel spherical projectiles showed a $90^{\circ}$ deflection angle.

Most of the steel projectiles tested for deflection force showed positive ferromagnetism (7 [63.6\%] of 11), with forces ranging from 514 to 444,000 dynes; the other $4(36.4 \%)$ were negative. Longitudinal migration was evident in 5 (71.4\%) of 7 steel projectiles tested, and absent in all nonsteel projectiles tested (59 [100\%]).

Heating was measured in 16 bullets placed inside a gelatinous material, which was comparable with soft tis- sue, and MR imaging was timed from 15 to 60 minutes. Bullet heating ranged from $1.6^{\circ} \mathrm{C}$ to $1.7^{\circ} \mathrm{C}$ (background heating of $1.5^{\circ} \mathrm{C}$ ), with an overall bullet heating range of $0.1^{\circ} \mathrm{C}-0.2^{\circ} \mathrm{C}$ for the 6 bullets tested in 2 of the studies. ${ }^{5,12}$ Smith et al. reported their results in Fahrenheit, with an overall increase of $2^{\circ} \mathrm{F}$ (range $1^{\circ} \mathrm{F}-4^{\circ} \mathrm{F}$ ) in 10 bullets..$^{22}$

Of the 9 steel bullets tested for artifact, 4 (44.4\%) produced severe artifact on MR images, and the remaining $5(55.6 \%)$ produced moderate artifact. Nonsteel projectiles produced no evident artifact or mild artifact in 39 $(88.6 \%)$ of the 44 scans with tested bullets; moderate artifact in 3 (6.8\%) of 44 (1 of which was of foreign origin, with a lead core/copper-nickel jacket); and severe artifact in $2(4.6 \%)$ of 44 (both of foreign origin, with lead core/ copper-nickel jackets [Table 2]).

A new ferromagnetic detection system that was used to screen for potential metallic implants was described by Karacozoff et al. ${ }^{12}$ It successfully uses a magnetometer (ferromagnetic sensor) that provides whole-body coverage; the metallic object must be in motion to detect its magnetic field. They placed multiple individuals, each holding a steel bullet in hand, in front of the detector, and all bullets tested positive for ferromagnetism.

\section{Clinical Studies}

A total of 22 patients described in 4 articles ${ }^{2,6,16,23}$ had a bullet or bullet fragment lodged near or in the spinal canal, and all underwent MR imaging studies. The number of patients per study ranged from 1 to 19 . The field strength of the MR scanner ranged from 1 to $3 \mathrm{~T}$. Clinical indications included suspicion of osteomyelitis $(n=19),{ }^{23}$ a pregnant woman with Brown-Séquard syndrome $(\mathrm{n}=$ $1),{ }^{6} \mathrm{SCI}$ without radiological abnormality $(\mathrm{n}=1),{ }^{2}$ and intradural migration of a bullet $(n=1) .{ }^{16}$

Neurological deficits included complete SCI $(\mathrm{n}=$ 18), ${ }^{16,23}$ incomplete SCI $(\mathrm{n}=3)^{2,23}$ and the previously mentioned right Brown-Séquard syndrome $(\mathrm{n}=1){ }^{6}$ The bullet or fragment was lodged inside the spinal canal in 21 patients and outside the canal in 1 patient. Two patients had bullets located in the cervical spine, $, 6,164$ had bullets lodged in the lumbar spine,,$^{2,11,16}$ and in 16 patients the location ranged from $\mathrm{C}-4$ to $\mathrm{T}-12 .{ }^{23}$

All of the patients underwent MR imaging, with the time from injury to MR imaging ranging from 7 days to 24 months. During and after the MR imaging session, none of the authors noted any patient-reported discomfort or painlike symptoms, heating, paresthesias, or weakness, and physical examinations showed no neurological or physical deterioration. The MR imaging studies obtained before and after CT scans in 16 patients ${ }^{23}$ failed to show bullet rotation or longitudinal migration. The remaining 6 patients did not undergo control CT scanning.

After MR evaluation, the decision was made to perform surgical decompression in 2 patients. One patient with a complete SCI had strength improvement from $0 / 5$ preoperatively to $2 / 5$ postoperatively. ${ }^{16}$ Another patient had significant neurological improvement, as reported by the authors but without further data described. ${ }^{6}$

Additionally, 2 more articles discuss clinical experiences with MR imaging and patients with spinal GSWs. Both were excluded from our clinical analysis, because 
Magnetic resonance imaging in gunshot wounds to the lumbar spine

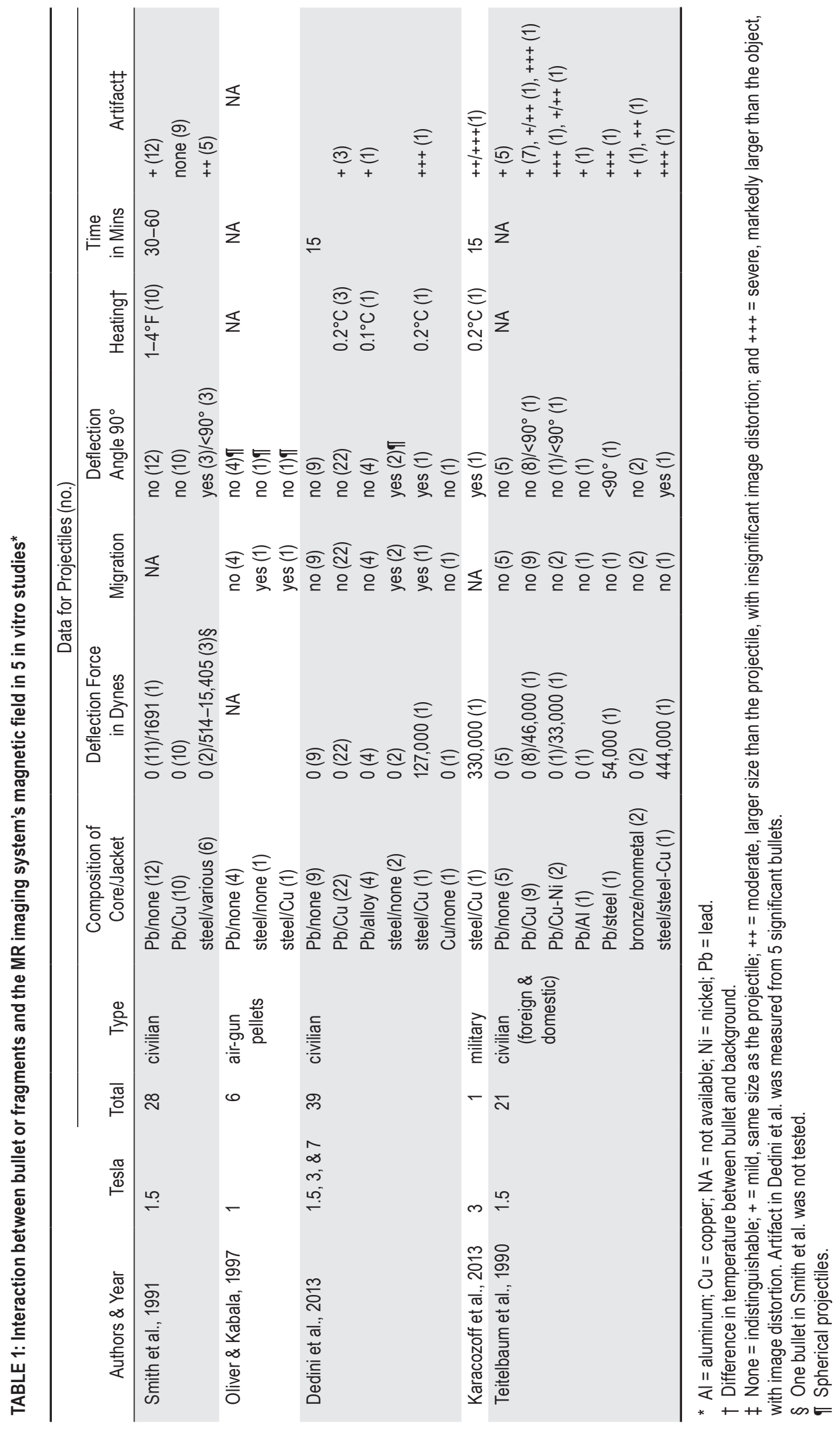




\section{E. Martinez-del-Campo et al.}

TABLE 2: Projectile artifact

\begin{tabular}{lrrccc}
\hline \multirow{2}{*}{$\begin{array}{c}\text { Bullet } \\
\text { Composition } \\
\text { (core/jacket) }\end{array}$} & No. & \multicolumn{4}{c}{$\begin{array}{c}\text { No. of Projectiles w/ Artifact } \\
\text { (\% of composition) }\end{array}$} \\
\cline { 3 - 6 } & None & + & ++ & +++ \\
\hline $\mathrm{Pb} /$ nonmetal & 19 & & $19(100)$ & & \\
\hline $\mathrm{Pb} / \mathrm{Cu}$ or Cu-Ni & 23 & $9(39.1)$ & $10(43.5)$ & $2(8.7)$ & $2(8.7)$ \\
bronze/nonmetal & 2 & & $1(50)$ & $1(50)$ & \\
\hline $\mathrm{Pb} /$ steel & 1 & & & & $1(100)$ \\
steel/various & 5 & & & $5(100)$ & \\
steel/Cu & 3 & & & & $3(100)$ \\
total (\% of total) & 53 & $9(17.0)$ & $30(56.6)$ & $8(15.1)$ & $6(11.3)$ \\
\hline
\end{tabular}

* See Table 1 for definitions of artifact categories.

they lacked sufficient data or reported that their data were anecdotal. In summary, a letter from Finitsis et al. ${ }^{8}$ reported on 19 patients with retained metallic fragments, presumed by the authors to have lead composition, in proximity to the spine or in the spinal canal. According to the authors, none of the patients experienced any adverse effects from MR imaging. Teitelbaum et al. ${ }^{25}$ reported on 7 patients who underwent MR imaging at 0.5 or $1.5 \mathrm{~T}$. These patients had bullets, shotgun pellets, or shrapnel fragments lodged in the following areas: intra- and paraspinous ( 2 patients), extremities ( 2 patients), intramyocardial (1 patient), intrathoracic (1 patient), and intracranial (1 patient). The authors found mild metal artifact on $85.7 \%$ of the patients' MR images, and only the intracranial shrapnel caused severe black void artifact, obscuring the image. However, the authors reported no discomfort or neurological or vascular deterioration in any patient.

\section{Discussion}

\section{Bullet Properties}

Most domestic bullets and shotgun pellets are generally nonferromagnetic, ${ }^{5,23,25}$ but this is not universally true. Bullet cores are most often composed of lead, although lead may be combined with other metals to confer a desired hardness. ${ }^{4}$ Foreign-origin projectiles may have impurities, which contain ferromagnetic metals. ${ }^{5,25}$ Additionally, bullets can be fully jacketed, nonjacketed, or partially jacketed. A "jacket" is a metallic layer over the bullet's surface, which may consist of steel, austenitic steel (nonmagnetic), copper, brass, or nickel; 4 these layers may provide additional ferromagnetic properties that must be taken into consideration when conducting imaging studies.

The bullet's potential movement caused by the magnetic field of the MR scanner is directly related to its ferromagnetism, mass, location, orientation, shape, and relation to the dura mater. ${ }^{5}$ Heating is related to the metallic composition, conductivity, mass, length, shape, distance to coil, field strength, and sequencing parameters of the MR scanner. ${ }^{5,23}$ Aluminum, titanium, and stainless steel have unremarkable internal and external heating, regardless of size. Small steel and copper fragments have negligible effects. ${ }^{23}$

\section{In Vitro Studies With MR Imaging and Bullets}

Throughout the last 2 decades, several in vitro studies have tested the effects that magnetic fields produce on different types of bullets, with 1.5-, 3-, and more recently 7-T MR scanners. ${ }^{5,12,16,22,25}$ Authors of these studies considered the ferromagnetic properties of 95 commonly used bullets and pellets, measuring (if any) their deflection force and angle, longitudinal migration, image artifact, and heating. However, further clinical data are necessary to reach accurate conclusions on the use of MR imaging in these patients.

Migration and Rotation. Although longitudinal migration was not noted in any of the nonsteel projectiles, it was evident in 5 (71.4\%) of 7 steel projectiles. The latter tend to deform or fragment on impact, increasing the surface area of the bullet tip, making predictions for potential displacement very difficult. ${ }^{23}$ Spherical bullets (BBs and shotgun pellets) lack a rotational axis, and therefore might have greater longitudinal migration, because they do not need to overcome magnetic torque to move. Oblong bullets rotate to align themselves to the long axis of the magnetic field, maximizing magnetic torque when oriented perpendicular to the field. This property makes rotation null when the bullet's long axis is oriented parallel to the $\mathrm{z}$ axis of the MR scanner. ${ }^{23,25}$ Despite this fact, $2(50 \%)$ of 4 steel spherical bullets showed a deflection angle of $90^{\circ}$.

Deflection force was evident in 7 (63.6\%) of 11 of the steel projectiles and in $3(3.9 \%)$ of 77 of the nonsteel projectiles tested, whereas $12(85.7 \%)$ of the 14 steel projectiles and $2(2.5 \%)$ of the 81 nonsteel projectiles tested showed some degree of rotation. The 3 nonsteel bullets that showed deflection force or rotation were all of foreign origin, supporting the belief that bullets not made in the US might contain ferromagnetic impurities. ${ }^{5,25}$ Overall, $10(11.4 \%$ of 88$)$ and 14 (14.7\% of 95) of all tested projectiles showed significant deflection force or rotation, respectively.

Heating. Overheating of the projectile and the surrounding tissue might be hazardous, especially if it is lodged near or in the spinal canal. ${ }^{23}$ However, a minimal increase $\left(0.2^{\circ} \mathrm{C}^{5}\right.$ and $\left.1^{\circ} \mathrm{F}-4^{\circ} \mathrm{F}^{22}\right)$ in the bullet's temperature above the surrounding tissue's temperature in extreme radiofrequency environments was considered clinically insignificant. When the bullets were relatively short $(16-19.5 \mathrm{~mm})$, the effect was even less clinically significant. ${ }^{5}$ Most authors concluded that heating represents a lesser danger than displacement, and is negligible in most cases. ${ }^{20}$

Artifact. Magnetic objects tend to distort the MR image because they manipulate the magnetic field by strong off-resonances, creating what is commonly referred to as artifact, which is visible as signal voids (black areas) or pileups (bright areas). ${ }^{1}$ An artifact is related to a material's susceptibility, dimensions, static magnetic field strength, and imaging parameters. ${ }^{5,19}$ In the study by Dedini et al., ${ }^{5}$ of 5 bullets tested for artifacts, the 4 without steel cores exhibited insignificant black voids, whereas a single steelcore bullet showed a very large signal void. All 9 steel 


\section{Magnetic resonance imaging in gunshot wounds to the lumbar spine}

bullets that were tested had significant (moderate to severe) artifact, whereas $39(88.6 \%)$ of the 44 nonsteel bullets tested had insignificant artifact.

\section{Management of GSWs}

Gunshot wounds in the spine may cause fractures or contusion of the vertebral body, with spinal cord and nerve root compression caused by bone or bullet fragments. ${ }^{2}$ The management of these wounds is based on the stability of the spine, the degree of SCI, and associated injuries. Neurosurgical procedures are not often required. ${ }^{17,26}$ Surgical spinal decompression with bullet/ fragment removal may not be necessary when complete SCI is evident on physical examination. Surgery plays a major role in treatment of patients with spinal instability, who require immediate fixation and arthrodesis, with different types of instrumentation tailored for each case. ${ }^{17}$ However, instability in the lumbar spine secondary to a GSW is rare. ${ }^{9}$ Potential late complications of GSW to the spine include wound infections, osteomyelitis, and (rarely) lead intoxication. ${ }^{20}$ All patients should have at least 6 months of outpatient follow-up to rule out infection before being discharged from care..$^{9,18}$ The potential risk of lead intoxication might lead to surgical removal of the bullet, as previously reported. ${ }^{17}$

\section{Diagnostic Imaging}

After hemodynamic stabilization is attained in a patient with a GSW, potential vertebral fractures and the location of the projectile must be determined using lateral and anteroposterior radiographs, with further confirmation using CT imaging, to establish what additional therapy may be needed. ${ }^{2,4,15}$ When vertebral fractures are not evident, instability at individual or multiple spinal segments can be assessed with dynamic radiographs of the spine, if needed. ${ }^{4}$

Role of $C T$. If spinal instability or neurological compression with progressive deterioration is evident in patients with spinal GSWs, immediate, accurate imaging is required. ${ }^{4} \mathrm{~A} \mathrm{CT}$ scan provides excellent bone resolution, but is inaccurate in determining the degree and extent of neurological injury. ${ }^{2,23}$

Role of MR Imaging. Previously, MR imaging has had almost no role in the acute management of SCI caused by GSWs, ${ }^{17,20,23}$ but it may be required in specific cases to evaluate neurological damage, after careful assessment of the type of projectile and its proximity to the spinal canal. ${ }^{4}$ During follow-up, MR imaging might be also required for better appraisal of improvement or exacerbation of the injury, and it is very useful in identifying ligamentous or disc disruption, which may cause spinal instability. ${ }^{23}$ The make and caliber of the projectile must be established by forensic data, relying on spent cartridges from crime scenes. ${ }^{23}$ This is the most important step in deciding if MR imaging should be performed in a patient with a bullet or fragment lodged in the lumbar spine. Unfortunately, at present this decision relies on forensic data, which may not be available, especially in emergency cases, because no specific radiographic (CT or x-ray) findings have been described that differentiate between ferromagnetic and nonferromagnetic projectiles. However, the magnetometer described by Karacozoff et al. ${ }^{12}$ might provide an immediate and safe screening method to establish the projectile's ferromagnetism, when used together with forensic information.

Magnetic resonance imaging provides markedly less artifact and better imaging of soft tissue and neural elements compared with any other type of imaging. ${ }^{2,6}$ To assess possible spinal infections during follow-up in patients with GSWs, MR imaging has been shown to have superior detail and accuracy, and is safe enough to be performed at least 2 months after the initial insult. ${ }^{9,18,23} \mathrm{Ar}-$ tifacts ranging from imperceptible to moderate are seen on MR images with nonferromagnetic bullets, whereas severe black void artifact and distortion may be caused by ferromagnetic bullets. This may be decreased with fat suppression, minimal echo times, fast spin echo, and wide bandwidth. ${ }^{25}$

Historically, when the bullet is located inside the spinal canal, it has been considered an absolute contraindication for MR imaging. ${ }^{23}$ However, nonjacketed and nonferromagnetic bullets may be considered safe enough to allow patients to undergo an MR study, even when the bullets or fragments are located inside the spinal canal. $5,12,16,25$ The clinical results we pooled in this analysis support this statement; however, in vitro results may raise concerns because of the small percentage of nonferromagnetic bullets that presented deflection and rotation under magnetic fields. The ferromagnetic detection system described by Karacozoff et al. ${ }^{12}$ could have a huge impact in the management of these patients; however, further studies are needed. ${ }^{5}$

\section{Clinical Experience With MR Imaging for Patients With GSWs}

Many reports of the use of MR imaging after a GSW to the spine have not supported the concern about causing bullet migration and possible dislodgment..$^{2,6,8,23,25}$ In 1999, Smugar et al. ${ }^{23}$ reported on 19 patients with bullets/ fragments located in proximity to the spinal cord who underwent MR imaging (1.5 T, multiple protocols) 2-24 months after initial injury, for suspicion of osteomyelitis. The bullet's position was pinpointed with CT imaging pre- and post-MR testing. In these authors' experience, none of the patients reported pain, warmth, or discomfort, and none suffered any neurological or physical deterioration. Furthermore, none had evidence of bullet migration or rotation. Teitelbaum et al. ${ }^{25}$ performed MR imaging in 7 patients who had retained bullets and shotgun pellets; all of them were safely imaged. Nonetheless, several authors, including Teitelbaum et al., recommend caution in using MR imaging when metallic foreign bodies are located near vital neural, vascular, or soft-tissue structures. ${ }^{5,23,25}$

Similar results have been found by other authors, in cases in which MR imaging aided in the decision to conduct surgical decompressive procedures. ${ }^{8}$ Surgery can be beneficial in patients with incomplete $\mathrm{SCI}^{3,10}$ in which neurological impingement is suspected of producing progressive neurological deterioration. ${ }^{26}$ In our literature sur- 


\section{E. Martinez-del-Campo et al.}

vey there was a report of 1 pregnant patient with a GSW to the upper cervical spine who experienced BrownSéquard syndrome and had multiple imaging studies, all failing to show the bullet's location and its relation to the spinal cord. However, the bullet was accurately shown on the MR image, without significant artifact or untoward effects. ${ }^{6}$ Another study included a patient with a GSW to the thoracolumbar spine and SCI without radiological abnormality. An immediate MR image showed swollen conus medullaris. ${ }^{2}$ Kafadar et al. ${ }^{11}$ also reported on a patient with a GSW to the spinal canal in the lumbar region in whom an MR image was obtained and who subsequently underwent surgical decompression. According to these authors and others, ${ }^{6,16}$ follow-up imaging in patients with spinal GSWs showed no migration or dislodging of the bullet in any case; none of the patients had any neurological deterioration; curiously, 2 of them experienced significant neurological improvement.

\section{Safety of MR Imaging}

The potential hazards, contraindications, and safety of MR imaging have been established in clinical and in vitro studies for several biomedical metallic implants. ${ }^{23}$ Variables such as tissue scarring, tissue resistance against displacement, and bullet fragmentation may significantly decrease the overall effect of the magnetic field on bullets embedded in tissue or bone. ${ }^{5}$ The results obtained in our analysis provide similar data to those already published. When clinical presentation and bullet composition is ideal in specific and carefully identified cases, MR imaging is a safe procedure, regardless of the location of the projectile in relation to the spinal canal. When a projectile is located in the lumbar spine, this procedure might be even safer than when it is located elsewhere along the spinal canal. Nerve roots below the conus medullaris are usually less prone to injury with minimal compression, and outcomes for patients with GSWs at or below the conus medullaris usually have a more favorable prognosis than for patients with SCIs above this level. ${ }^{13,20-22}$ Evidence from the 2 patients who underwent MR imaging shortly after injury and received surgical decompression shows that they experienced neurological improvement. ${ }^{6,16}$ This supports the view that MR imaging does not present even a minimal danger; it is actually beneficial for a patient when it orients the physician to accurate diagnosis and treatment.

Some authors of the in vitro studies we analyzed suggest that their results might be reproducible in clinical scenarios, and they recommend that metallic fragments located inside the spinal canal should represent an absolute contraindication for MR imaging. ${ }^{5}$ As evidenced in the literature and in the results we present of in vitro and clinical scenarios, this belief appears to be refutable. ${ }^{2,6,8,23}$ Further clinical data in patients with incomplete SCI and with bullets in the spinal canal might reinforce this position.

\section{Limitations of the Study}

This is a systematic review of the literature, based on information dating from 1985 to 2014 . Results presented in this paper may not be reproducible in different clinical or radiological scenarios. No long-term follow-ups were reported in the articles included in the review. Overexposure of metallic objects to the scanner's magnetic field might present unexpected results in clinical scenarios.

\section{Conclusions}

Magnetic resonance imaging is the best imaging tool to evaluate neurological structures and soft tissue, and to document the extent of neurological injury and infection. Metallic implants represent a relative contraindication to MR scans, and the decision to obtain an MR image for any patient with a GSW near the spine should be made only after careful analysis of the patient's specific presentation, assessment of risks versus benefits, comprehensive and thorough discussion with the patient, and understanding of the interactions between the magnetic field and the specific projectile in question. Often, the only symptom (if any) ever reported by patients with bullets/fragments who are undergoing an MR scan is mild discomfort from a heating sensation; this symptom should lead to early abandonment of the study. However, none of the analyzed data showed that patients with GSWs undergoing MR imaging experienced any discomfort. Even though ferromagnetic materials inside the spinal cord represent an absolute contraindication for MR imaging, the potential risk of injury from MR imaging for reassessment of neurological status or infection might be significantly decreased 2 months after a GSW or similar injury. In acute settings, when incomplete SCI is evident without radiological confirmation of injury by using conventional imaging studies, MR imaging might be the only diagnostic tool accurate enough to establish the degree of damage, and to support a decision on whether to perform surgical decompression.

\section{Disclosure}

The authors report no conflict of interest concerning the materials or methods used in this study or the findings specified in this paper.

Author contributions to the study and manuscript preparation include the following. Conception and design: Martinez-del-Campo, Rangel-Castilla, Soriano-Baron. Acquisition of data: Martinez-delCampo. Analysis and interpretation of data: Martinez-del-Campo. Drafting the article: Martinez-del-Campo, Rangel-Castilla. Critically revising the article: Theodore, Soriano-Baron. Reviewed submitted version of manuscript: Theodore. Statistical analysis: Martinez-delCampo. Study supervision: Theodore.

\section{References}

1. Bachschmidt T, Lipps F, Nittka M: syngo WARP - Metal artifact reduction techniques in magnetic resonance imaging. MAGNETOM Flash 2:24-25, 2012

2. Bashir EF, Cybulski GR, Chaudhri K, Choudhury AR: Magnetic resonance imaging and computed tomography in the evaluation of penetrating gunshot injury of the spine. Case report. Spine (Phila Pa 1976) 18:772-773, 1993

3. Benzel EC, Hadden TA, Coleman JE: Civilian gunshot wounds to the spinal cord and cauda equina. Neurosurgery 20:281285, 1987

4. Bono CM, Heary RF: Gunshot wounds to the spine. Spine J 4:230-240, 2004

5. Dedini RD, Karacozoff AM, Shellock FG, Xu D, McClellan 


\section{Magnetic resonance imaging in gunshot wounds to the lumbar spine}

RT, Pekmezci M: MRI issues for ballistic objects: information obtained at 1.5-, 3- and 7-Tesla. Spine J 13:815-822, 2013

6. Ebraheim NA, Savolaine ER, Jackson WT, Andreshak TG, Rayport M: Magnetic resonance imaging in the evaluation of a gunshot wound to the cervical spine. J Orthop Trauma 3: 19-22, 1989

7. Eshed I, Kushnir T, Shabshin N, Konen E: Is magnetic resonance imaging safe for patients with retained metal fragments from combat and terrorist attacks? Acta Radiol 51:170-174, 2010

8. Finitsis SN, Falcone S, Green BA: MR of the spine in the presence of metallic bullet fragments: is the benefit worth the risk? AJNR Am J Neuroradiol 20:354-356, 1999

9. Heary RF, Vaccaro AR, Mesa JJ, Balderston RA: Thoracolumbar infections in penetrating injuries to the spine. Orthop Clin North Am 27:69-81, 1996

10. Isiklar ZU, Lindsey RW: Gunshot wounds to the spine. Injury 29 (Suppl 1):SA7-SA12, 1998

11. Kafadar AM, Kemerdere R, Isler C, Hanci M: Intradural migration of a bullet following spinal gunshot injury. Spinal Cord 44:326-329, 2006

12. Karacozoff AM, Pekmezci M, Shellock FG: Armor-piercing bullet: 3-T MRI findings and identification by a ferromagnetic detection system. Mil Med 178:e380-e385, 2013

13. Kitchel SH: Current treatment of gunshot wounds to the spine. Clin Orthop Relat Res (408):115-119, 2003

14. Kumar R, Lerski RA, Gandy S, Clift BA, Abboud RJ: Safety of orthopedic implants in magnetic resonance imaging: an experimental verification. J Orthop Res 24:1799-1802, 2006

15. Moon E, Kondrashov D, Hannibal M, Hsu K, Zucherman J: Gunshot wounds to the spine: literature review and report on a migratory intrathecal bullet. Am J Orthop 37:E47-E51, 2008

16. Oliver C, Kabala J: Air gun pellet injuries: the safety of MR imaging. Clin Radiol 52:299-300, 1997

17. Rentfrow B, Vaidya R, Elia C, Sethi A: Lead toxicity and management of gunshot wounds in the lumbar spine. Eur Spine $\mathbf{J}$ 22:2353-2357, 2013
18. Romanick PC, Smith TK, Kopaniky DR, Oldfield D: Infection about the spine associated with low-velocity-missile injury to the abdomen. J Bone Joint Surg Am 67:1195-1201, 1985

19. Shellock FG: Biomedical implants and devices: assessment of magnetic field interactions with a 3.0-Tesla MR system. J Magn Reson Imaging 16:721-732, 2002

20. Shellock FG, Crues JV: High-field-strength MR imaging and metallic biomedical implants: an ex vivo evaluation of deflection forces. AJR Am J Roentgenol 151:389-392, 1988

21. Shellock FG, Curtis JS: MR imaging and biomedical implants, materials, and devices: an updated review. Radiology 180:541-550, 1991

22. Smith AS, Hurst GC, Duerk JL, Diaz PJ: MR of ballistic materials: imaging artifacts and potential hazards. AJNR Am J Neuroradiol 12:567-572, 1991

23. Smugar SS, Schweitzer ME, Hume E: MRI in patients with intraspinal bullets. J Magn Reson Imaging 9:151-153, 1999

24. Spakauskas B, Ambrozaitis KV, Kontautas E: The bullet in the dural sac. How to catch it? A report of two cases. Medicina (Kaunas) 43:478-481, 2007

25. Teitelbaum GP, Yee CA, Van Horn DD, Kim HS, Colletti PM: Metallic ballistic fragments: MR imaging safety and artifacts. Radiology 175:855-859, 1990

26. Waters RL, Sie IH: Spinal cord injuries from gunshot wounds to the spine. Clin Orthop Relat Res (408):120-125, 2003

Manuscript submitted March 13, 2014.

Accepted July 11, 2014.

Please include this information when citing this paper: DOI: 10.3171/2014.7.FOCUS1496.

Address correspondence to: Nicholas Theodore, M.D., c/o Neuroscience Publications, Barrow Neurological Institute, St. Joseph's Hospital and Medical Center, 350 W. Thomas Rd., Phoenix, AZ 85013.email: neuropub@dignityhealth.org. 